\title{
Development and Study of a Mucoadhesive Formulation with EGF and Hyaluronic Acid for Gum Application in Periodontitis
}

\author{
Gabriella Russo ${ }^{1,2}$, Rocío Amate-Marchal ${ }^{2}$, Herminia Castán ${ }^{1}$, Elena Sánchez-Fernández ${ }^{3}$ and \\ Adolfina Ruiz-Martínez ${ }^{1 *}$ \\ ${ }^{1}$ Department of Pharmacy and Pharmaceutical Technology, School of Pharmacy, University of Granada, Spain \\ ${ }^{2}$ Department R\&D of Inves Biofarm, Spain \\ ${ }^{3}$ Department of Oral Surgery, School of Dentistry, University of Granada, Granada, Spain \\ *Corresponding author: Ruiz Martínez, Department of Pharmacy and Tecnological Pharmacy, Campus of Cartuja s/n 18071- \\ Granada, Spain
}

\section{ARTICLE INFO}

Received: March 19, 2020

Published: 慧 April 01, 2020

Citation: Gabriella R, R Amate-Marchal, Herminia C, E Sánchez-Fernández, A RuizMartínez. Development and Study of a Mucoadhesive Formulation with EGF and Hyaluronic Acid for Gum Application in Periodontitis. Biomed J Sci \& Tech Res 26(5)-2020. BJSTR. MS.ID.004413.

Keywords: Mucoadhesive; EGF; HA; Periodontitis

\section{ABSTRACT}

Backgroud: Periodontal treatments need for a better results mucoadhesive formulas. Polymeric excipients of mucoadhesive formulas attach to mucus allowing the delivery of actives ingredients. Evaluation of the mucous permeation and cession is essential to determinate topical formula stability, bioavailability and effectiveness.

Aim: This work has the aim to study the physical chemicals and organoleptic characteristics, stability, permeability and cession of a formulation with EGF and Hyaluronic acid for the application in gingival tissue.

Method: Three different formulas were developed with mucoadhesive polymer. $\mathrm{pH}$, viscosity and extensibility were measured during the time where formula was stressed to environmental conditions of temperature and humidity to study its stability and homogeneity in the time. Emulsion formula was elected like best formulation. Emulsion was studied with Franz cells to evaluate the EGF cession and permeation. Protein such us EGF contains aromatic groups with $275 \mathrm{~nm}$ absorbance peak. Protein also has absorbance peak at 220-230 nm due to its peptide bond.

Result: Comparing UV-VIS absorbance at different times point it decrease along time. For cession absorbance data for 24 hours could implicate protein is stable during this time. The date obtained show that the chicken pouch membrane had complicated compound than nylon membrane and EGF could interaction with its.

Conclusion: Mucoadhesion polymers provide appropriate extensibility and viscosity to increase active ingredient delivery and contact surface. Formulation design includes the evaluation and the screening of the stability and bioactivity efficacy. Due to laboratory results of different assays (viscosity, extensibility, stability, cession and permeation), emulsion was selected to use in periodontitis treatment for a pilot study.

\section{Short Communication}

Periodontal treatments include mucoadhesive formula such as films, gels, viscous solutions and spray [1]. Gingival tissue is covered by keratinized mucosa and mucus to protect it against physical and chemical damage [2]. Polymeric excipients of mucoadhesive formulas attach to mucus a glycoprotein polymer and create bonds between itself and biological membrane [3]. Junctions increase

retainment time and permeability of drug in gingival tissue. Hyaluronic acid (HA) has been uses in pharmaceutical industry [4] due to it biocompatibility, mucoadhesivity, macromolecular size, viscosity and hygroscopicity [5]. Recombinant human epidermal growth factor (rhEGF) haven been used in experimental and clinical investigation of dermatitis defects. EGF is a signalling peptide 
present in mammalian stimulating cell growth, proliferation and differentiation. EGF ligand-receptor binding activates tyrosine kinase cascade increasing intracellular calcio, glycolysis, gene expression and protein synthesis enhancing cells motility and adhesiveness [6]. It is essential to manteain homeostatic balance between epithelial cell proliferation and differentiation [7]. The aim of this work was to design, perform and evaluate a mucoadhesive topical formula to improve periodontitis defects. Actives ingredients were hyaluronic acid and EGF. Hyaluronic acts

Table 1: Three formulas composition. as EGF transport. The three formulas with different mucoadhesive polymers were designed and analysed its stability and extensibility. The efficacy of selected formula to treat periodontitis was evaluated in a randomized controlled clinical pilot study [8].

\section{Materials and Methods}

\section{Semisolid Formulations}

Three topical formulation were designed hydrogel, emulsion and liposome emulsion (Table 1).

\begin{tabular}{|c|c|c|c|c|c|}
\hline \multicolumn{2}{|c|}{ Emulsion } & \multicolumn{2}{c|}{ Hydrogel } & \multicolumn{2}{c|}{ Liposome Emulsion } \\
\hline Ingredients & Composition (\%) & Ingredients & Composition (\%) & Ingredients & Composition (\%) \\
\hline Hyaluronic acid & $0,5-1$ & Hyaluronic acid & $0,5-1$ & Hyaluronic acid & $0,5-1$ \\
\hline EGF oil & $0,5-1$ & PBS with EGF & $0,5-1$ & EGF oil & $0,5-1$ \\
\hline Carbomer & $0,5-1$ & Sorbitol 70\% & $40-50$ & PBS with EGF & 6 -May \\
\hline CMC & $0,5-1$ & Povidone & $0,5-1$ & CMC & $0,5-1$ \\
\hline Glycerine & $20-0 c t$ & Propanediol & $5-$ Apr & Carbomer & $0.4-0.5$ \\
\hline $\begin{array}{c}\text { Hydroxypropyl- } \\
\text { methylcellulose }\end{array}$ & $0,5-1$ & Xanthan Gum & $0,5-1$ & Citric Acid & $0,5-1$ \\
\hline Citric acid & $0,1-0,2$ & EDTA & $0.04-0.05$ & Sodium citrate & $0.1-0.2$ \\
\hline Sodium citrate & $0,2-0,3$ & Citric Acid & $0.1-0.2$ & LIPOSYSTEM & COMPLEX \\
\hline Tween 80 & $2-J a n$ & Sodium citrate & $0.2-0.3$ & Sodium Benzoate & $2-0.3$ \\
\hline Sodium Benzoate & $0.1-0.3$ & Sodium Benzoate & $0.1-0.3$ & Aqua & cs 100 \\
\hline Aqua & cs 100 & Aqua & cs 100 & \\
\hline
\end{tabular}

\section{Previous Stability}

Homogeneity of formulas was determinate by centrifugation test. Physical properties are kept constant in stable formulas. Samples were warmed up to 40 OC and centrifugated at 3000 rpm during 30 minutes. (Hermle, model: Z36HK). Extensibility measure semi-solid formulation surface covering. Extensometer apply pressure to $300 \pm 0.200 \mathrm{mg}$ of sample. Stability of emulsion was analysed by cold/heat cycles assay. pH, viscosity organoleptic properties were measure before and after process. Viscosity of formulas were measures with fungilab, modelSmart. The spindle used was R7 at $100 \mathrm{rpm}$. Viscosity were determinate through 3 measurements. Cession and mucosal permeation of EGF were measured by diffusion cells process with Franz Cells [9].

\section{Result and Discussion}

Organoleptic properties, previous stability and extensibility were measured for three formulations. Emulsion final formula was white with moderate viscosity, sticky and adhesively. Liposome emulsion was yellow, not sticky and greasy. Hydrogel was transparent viscosity, soft and fresh. Emulsion, liposome emulsion and hydrogel final formula kept homogeneity after previous stability test. Extensibility determines availability and efficacy of topical formula active ingredients. High extensibility improves local active ingredients delivery and mucoadhesive polymers attachment to gingival tissue. Hydrogel and liposome emulsion had similar extensibility. Emulsion extensibility is higher than other formula. It was determinate with aspect, $\mathrm{pH}$ and viscosity parameter of emulsion. Initial $\mathrm{pH}$ was 5.95 for emulsion, 5.68 for liposomated emulsion and 5.53 for hydrogel. Viscosity (mPas) was 16401 for emulsion, 30932 for liposomated emulsion and 6789 for hydrogel. Organoleptic properties were stable during the process. $\mathrm{pH}$ mean was $5.9 \pm 0.05$ without significative variation at different times. Aspect and pH homogeneity show stable formulation. Based on laboratory results emulsion semi-solid formulation was select. Cession and mucosal permeation were evaluated with Franz cells system.

Cell diffusion system indicates the effectiveness of semi-solid formulation to release its active ingredients to be transport to mucosa. Aliquots from receptor compartment were collected at three times (30 minutes, 3 hours and 24 hours) and analysed by UV-VIS absorbance. This work evaluated the EGF cession and permeation included in emulsion formula. Aromatic side-chains residues (Trp, Tyr and Phe) of proteins surface has UV absorbance in 240-300nm region [10]. EGF surface has tyrosine residues (Tyr13 and Tyr37) [11,12], that may have an absorbance peak at $275 \mathrm{~nm}$ when $\mathrm{pH}$ is neutral. Thus, we supposed UV-VIS absorbance peak of EGF was $275 \mathrm{~nm}$. EGF (10 ppm) ultraviolet spectrum (Figure 1) showed low absorbance intensity at $275 \mathrm{~nm}$ and high peak at 220 $\mathrm{nm}$. Lower peak at $275 \mathrm{~nm}$ may be because low concentration of protein and low absorbance power of tyrosine. High peak at $220 \mathrm{~nm}$ 
was not analysed. Equation was $\mathrm{Y}=0.0009 \mathrm{X}+0.0265$. Regression Concentration for EGF in two assays cession and permeation was line was accurate based on regression coefficient $\left(R^{2}=0.9554\right)$. calculated with regression equation.

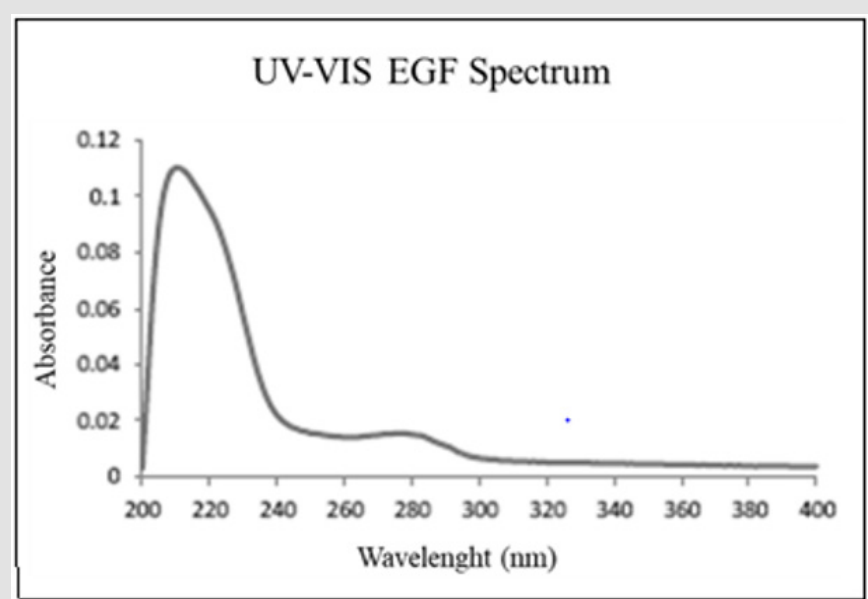

Figure 1: EGF absorbance spectrum (10 ppm).

Absorbance in both blank and emulsion aliquots was higher in chicken pouch membrane than nylon membrane. For nylon membrane, EGF concentration increase with time $(1.67 \cdot 105 \mathrm{ppm}$ at 24 hours and 2.31.104 at 24 hours) (Figure 2A). For chicken membrane after 3 hours concentration was (1.81.104) and at 24 hours (3.81.105) (Figure 2B). We hypothesized increasing of absorbance intensity at $240-260 \mathrm{~nm}$ range in cession and permeation spectrums could be related with denaturalization of EGF. Protein crossing through membrane could altered its protein structure and cysteine residues of its three internal disulphide bond would be exposed and had ultraviolet absorbance at $260 \mathrm{~nm}$ [12]. Nylon membrane spectrum has a higher absorbance range at 260-300 nm than chicken pouch membrane.

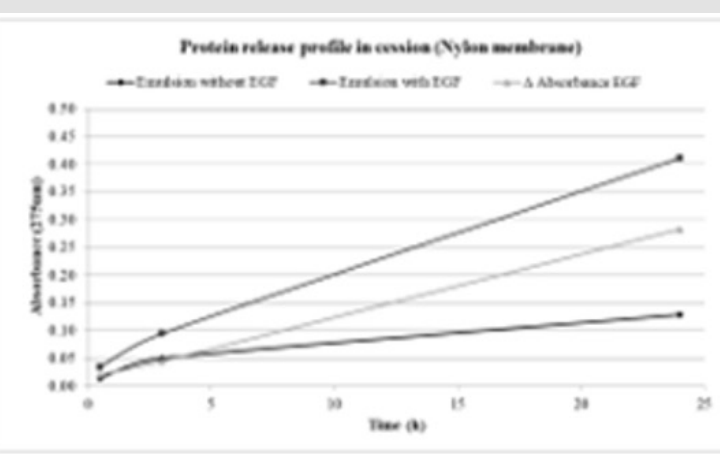

Figure 2A

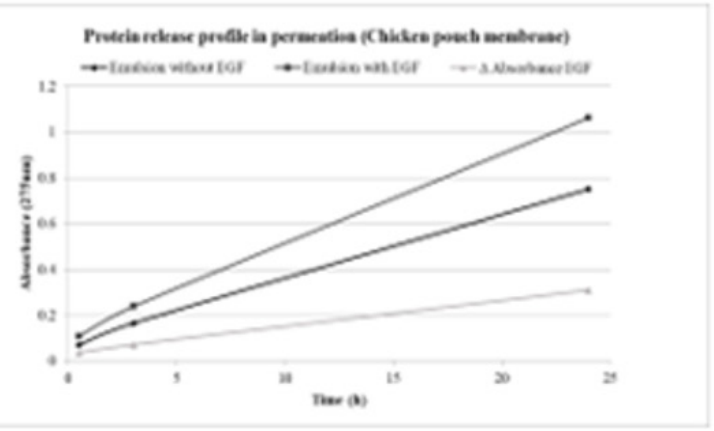

Figure 2B

Figure 2: A. Three point time aliquos absorbance at $275 \mathrm{~nm}$ in cession assay and

B. Permeation assay.

\section{Conclusion}

Physical and organoleptic properties of a semi-solid formulation are essential in formula designing. Mucoadhesion polymers provide appropriate extensibility and viscosity to increase active ingredient delivery and contact surface. Due to laboratory results of different assays (viscosity, extensibility and stability), emulsion was selected to use in periodontitis treatment.

\section{Conflicts of Interest}

The authors report no conflicts of interest related to the presented case. The company Inves Biofarm had facilitated the product for this study.

\section{Acknowledgment}

This study is a part of a PhD work in the Program of Pharmacy University of Granada.

\section{References}

1. Khutoryanskiy VV (2011) Advances in Mucoadhesion and Mucoadhesive Polymers. Macromol Biosci 11(6): 748-764.

2. Fonseca-Santos B, Chorilli M (2018) An overview of polymeric dosage forms in buccal drug delivery: State of art, design of formulations and their in vivo performance evaluation. Mater Sci Eng 86: 129-143.

3. Gandhi RB, Robinson JR (1994) Oral cavity as a site for bioadhesive drug delivery. Advanced Drug Delivery Reviews 13: 43-74.

4. Fallacara A, Baldini E, Manfredini S, Vertuani S (2018) Hyaluronic acid in the third millennium. Polymers 10: 701. 
5. Knopf-Marques H, Pravda M, Wolfova L, Velebny V, Schaaf P, et al (2016) Hyaluronic Acid and Its Derivatives in Coating and Delivery Systems: Applications in Tissue Engineering, Regenerative Medicine and Immunomodulation. Adv Healthc Mater 5(22): 2841-2855.

6. Esquirol Caussa J, Herrero Vila E (2015) Factor de crecimiento epidérmico, innovación yseguridad. Med Clin 145(7): 305-312.

7. Parkar MH, Kuru L, Giouzeli M, Olsen I (2001) Expression of growthfactor receptors in normal and regenerating human periodontal cells. Arch Oral Biol 46(3): 275-284.

8. Russo G, Sánchez J, González G, Gutiérrez R, Amate R, Sánchez E, et al (2020) Evaluation of the efficacy of EGF and hyaluronic acid formulation on periodontal defects named also black holes. Health Prim Care 4: 1-5.
9. Castán H, Ruiz MA, Clares B, Morales ME (2015) Design, development and characterization of buccal bioadhesive films of Doxepin for treatment of odontalgia. Drug Deliv 22(6): 869-876.

10. Crow J, Beckman JS (1995) Quantitation of Protein Tyrosine, 3-Nitrotyrosine, and 3-Aminotyrosine Utilizing HPLC and Intrinsic Ultrviolet Absorbance. Methods 7(1): 116-120.

11. Lu S, Chai JJ, Li M, Huang B-R, He CH, et al. (2001) Crystal Structure of Human Epidermal Growth Factor and Its Dimerization* He-Downloaded from. J Biol Chem 276(37): 34913-34917.

12. Brady P, Macnaughtan M (2015) Evaluation of colorimetric assays for analyzing reductively methylated proteins: Biases and mechanistic insights. Anal Biochem 491: 43-51.
ISSN: 2574-1241

DOI: $10.26717 /$ BJSTR.2020.26.004413

Adolfina Ruiz-Martínez. Biomed J Sci \& Tech Res

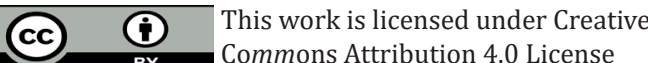

Submission Link: https://biomedres.us/submit-manuscript.php

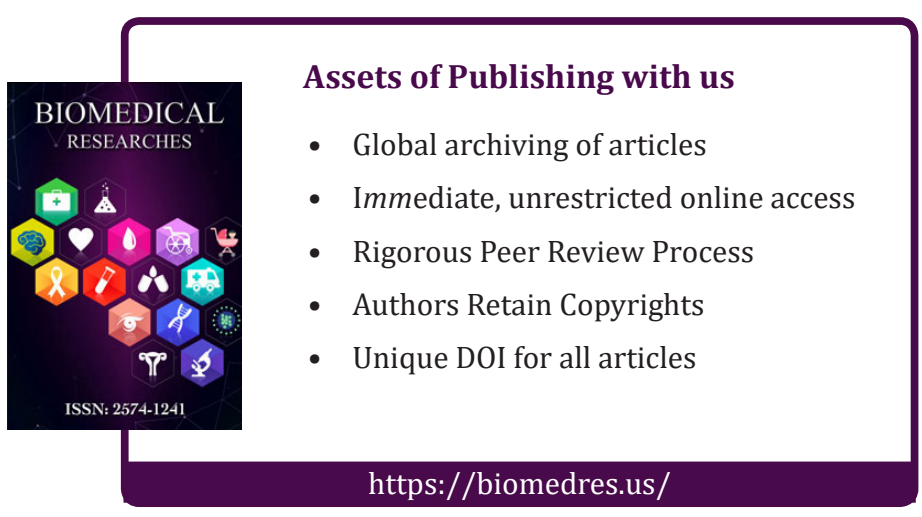

